\title{
Oral Dextrose Gel Reduces the Need for Intravenous Dextrose Therapy in Neonatal Hypoglycemia
}

\author{
Munmun Rawat Praveen Chandrasekharan Stephen Turkovich \\ Nancy Barclay Katherine Perry Eileen Schroeder Lisa Testa \\ Satyan Lakshminrusimha
}

Division of Neonatology, Department of Pediatrics, Women and Children's Hospital of Buffalo, Buffalo, N.Y., USA

\begin{abstract}
What Is It about?
Persistent asymptomatic hypoglycemia in newborns not responding to feeding often requires intravenous dextrose therapy. Previously, a randomized trial studied the use of $40 \%$ dextrose gel and feeds for asymptomatic hypoglycemia and showed reduction in the need for intravenous dextrose therapy with improved breastfeeding and maternal-infant bonding. We studied the impact of introducing a protocol for late-preterm/term asymptomatic hypoglycemic neonates incorporating supplementation of feeds with dextrose gel at a regional perinatal center in the United States. After implementation of the $40 \%$ dextrose gel, there was a reduction in intravenous dextrose therapy as well as reduced hospital costs and improved breastfeeding rates at discharge.
\end{abstract}

\section{Key Words}

Hypoglycemia · Dextrose gel

\begin{abstract}
Background: Newborn infants with risk factors may require intravenous (IV) dextrose for asymptomatic hypoglycemia. Administration of IV dextrose and transfer to the neonatal intensive care unit (NICU) may interfere with parent-infant bonding. Objective: To study the effect of implementing dextrose gel supplement with feeds in late preterm/term infants affected by asymptomatic hypoglycemia on reducing IV dextrose therapy. Method: A retrospective study was conducted before and after dextrose gel use: 05/01/2014 to 10/31/2014 and 11/01/2014 to 04/30/2015, respectively. Asymptomatic hypoglycemic (blood glucose level $<45 \mathrm{mg} / \mathrm{dl}$ ) infants in the newborn nursery (NBN) were given a maximum of 3 doses of dextrose gel ( $200 \mathrm{mg} / \mathrm{kg}$ of $40 \%$ dextrose) along with feeds. Transfer to the NICU for IV dextrose was considered treatment failure. Results: Dextrose gel with feeds increased the blood glucose level in 184/250 (74\%) of asymptomatic hypoglycemic infants compared to 144/248
\end{abstract}


(58\%) with feeds only $(\mathrm{p}<0.01)$. Transfer from the NBN to the NICU for IV dextrose decreased from $35 / 1,000$ to $25 / 1,000$ live births ( $p<0.01$ ). Exclusive breastfeeding improved from 19 to $28 \%(p=0.03)$. Conclusions: Use of dextrose gel with feeds reduced the need for IV fluids, avoided separation from the mother and promoted breastfeeding. Neonates who failed dextrose gel therapy were more likely to be large for gestational age, delivered by cesarean section and had lower baseline blood glucose levels.

(C) 2016 The Author(s)

Published by S. Karger AG, Basel

\section{Introduction}

Neonatal hypoglycemia is a global health problem and a preventable cause of neurological injury during the newborn period [1-3]. Approximately $15 \%$ of neonates are at risk for neonatal hypoglycemia [4]. Some of these infants fail to normalize their blood glucose level by feeds alone and require intravenous (IV) dextrose therapy. Preterm, small for gestational age (SGA) infants, infants of diabetic mothers and large for gestational age (LGA) infants are at high risk of requiring IV dextrose therapy due to persistent hypoglycemia. Many institutions, including ours, transfer neonates in need of IV dextrose from the newborn nursery (NBN) to the neonatal intensive care unit (NICU). This leads to separation of the baby from his/her mother, undue anxiety affecting bonding and breastfeeding, as well as contributing to increased hospitalization costs. Symptomatic infants with hypoglycemia require prompt glucose supplements preferably through the IV route to reduce symptoms and prevent longterm sequelae. However, a large number of 'at risk' neonates screened for hypoglycemia are asymptomatic and are treated due to the association between prolonged hypoglycemia and neurological impairment [3]. Approximately $10 \%$ of these asymptomatic neonates require admission to a NICU for the treatment of neonatal hypoglycemia adding an estimated USD 2.1 billion to health care costs annually in the United States [5]. Controversy still surrounds the definition, significance and management of neonatal hypoglycemia [6]. There is limited scientific evidence to guide treatment of asymptomatic neonatal hypoglycemia [4]. A blood glucose level maintained at $\geq 47 \mathrm{mg} / \mathrm{dl}$ is not associated with adverse neurological outcome and is generally considered a safe target after the first $4 \mathrm{~h}$ of postnatal age [3]. Among adults, $40 \%$ dextrose gel is used for treating hypoglycemia [7]. Harris and colleagues [8] conducted a randomized, double-blind, placebo-controlled trial of 230 infants (115 infants randomized to dextrose gel and 115 to placebo) and concluded that treatment with $40 \%$ dextrose gel along with breastfeeding, expressed breast milk or formula was more effective than feeds alone and increased blood glucose levels. They recommended that dextrose gel be considered for firstline management of late preterm and term hypoglycemic babies in the first $48 \mathrm{~h}$ after birth. A recent published meta-analysis had similar conclusions [8]. Dextrose gel is inexpensive (USD 12.99 for 3 tubes of $15 \mathrm{~g}$ each or approximately USD 1 per dose of 1,200 mg/3 ml) and can be prepared by the hospital pharmacy.

We implemented a protocol including dextrose gel therapy for asymptomatic hypoglycemic infants at $\geq 35$ weeks postmenstrual age (PMA). Our objectives were to determine whether feeds supplemented with $40 \%$ dextrose gel are more effective than feeds alone in treating asymptomatic neonatal hypoglycemia in term and late preterm neonates in the first $48 \mathrm{~h}$ after birth admitted to the NBN. We also evaluated the impact of this protocol on the number of NICU admissions for IV dextrose therapy. 
(c) 2016 The Author(s). Published by S. Karger AG, Basel www.karger.com/bmh

Rawat et al.: Oral Dextrose Gel Reduces the Need for Intravenous Dextrose Therapy in Neonatal Hypoglycemia

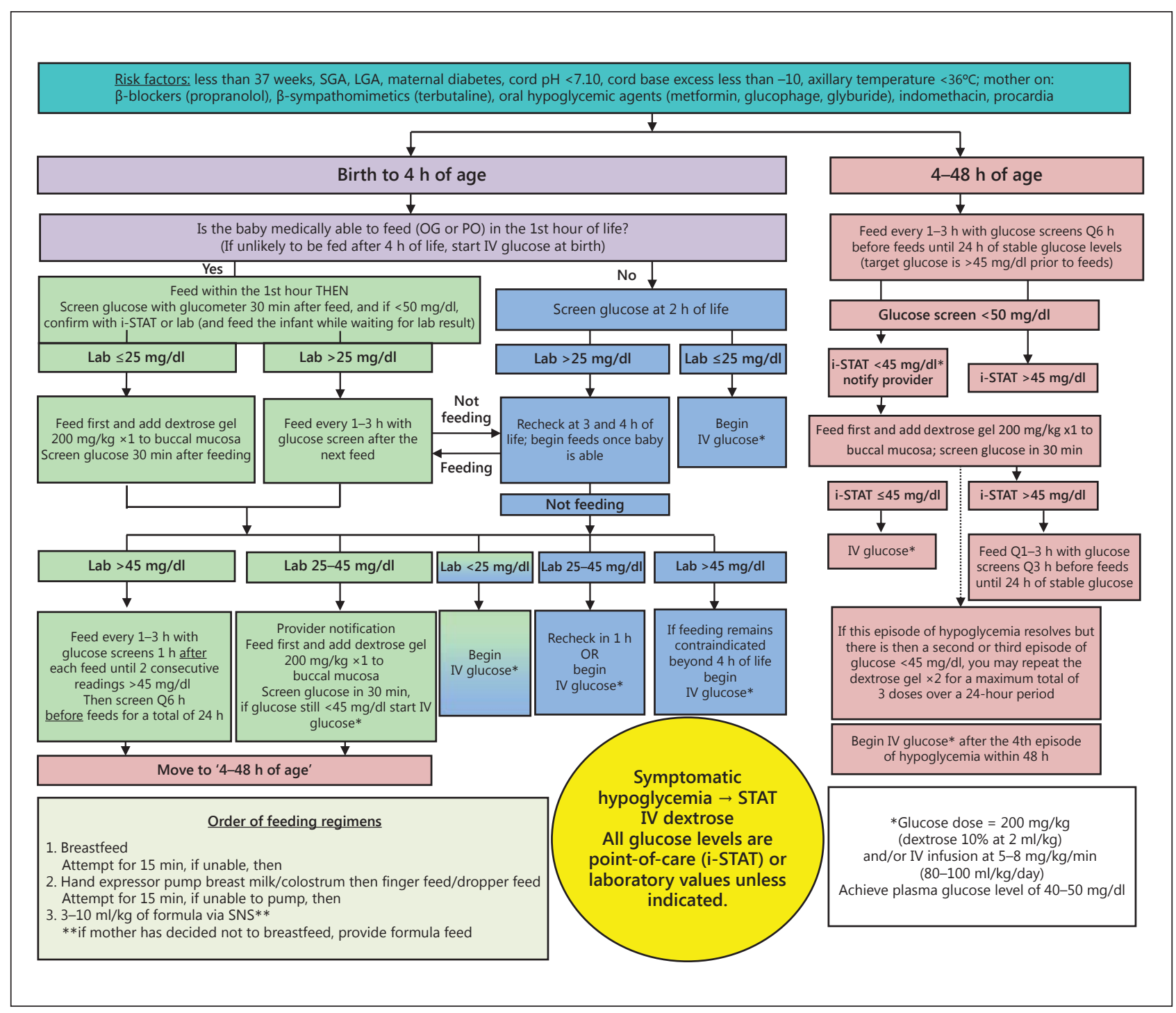

Fig. 1. Newborn nursery protocol for the screening and management of asymptomatic hypoglycemia. $O G=$ Orogastric; PO = per os; SNS = supplemental nursing system.

\section{Methods}

This retrospective chart review was approved by the Children and Youth Institutional Review Board at the University at Buffalo.

After a review of the existing literature on dextrose gel, a multidisciplinary committee added dextrose gel to the neonatal asymptomatic hypoglycemia protocol (fig. 1). Dextrose gel (40\%, Glutose $15^{\mathrm{TM}}$, Perrigo, Dublin, Ireland; ingredients: purified water, d-glucose USP $40 \%$, glycerin, lemon flavoring and Methylparaben NF in an oral gel base) was packaged into $1,200 \mathrm{mg} / 3 \mathrm{ml}$ doses in oral syringes and stocked in the Pyxis in the NBN. Weight-based dosing of dextrose gel was established with the help of the pharmacy $(200 \mathrm{mg} / \mathrm{kg}$ or $0.5 \mathrm{ml} /$ $\mathrm{kg}$ applied to buccal mucosa; online suppl. video file; for all online suppl. material, see www. karger.com/doi/10.1159/000448511). 
Mandatory educational sessions were provided to all nurses that focused on the use of the new algorithm (fig. 1) and the technique of administering the gel. This algorithm was implemented at the Regional Perinatal Center at the Women and Children's Hospital of Buffalo and at an affiliated nursery at Millard Fillmore Suburban Hospital. The use of 40\% dextrose gel was started in November 2014.

\section{Hypoglycemia Guidelines}

Two protocols were developed for the treatment of infants $\geq 35$ weeks PMA - one for symptomatic hypoglycemia (data not shown) and a second protocol for asymptomatic infants at risk for hypoglycemia. Figure 1 shows the protocol used in our institution to treat asymptomatic hypoglycemia.

A maximum of 3 doses of dextrose gel were given during the first $48 \mathrm{~h}$. If the blood glucose level was $<25 \mathrm{mg} / \mathrm{dl}$ after the administration of dextrose gel, the infant was admitted to the NICU for IV dextrose therapy. At any point, if the baby developed symptoms secondary to hypoglycemia, a STAT mini-bolus ( $2 \mathrm{ml} / \mathrm{kg}$ of $10 \%$ dextrose IV) was administered and an IV $10 \%$ dextrose infusion was initiated at $60-80 \mathrm{ml} / \mathrm{kg} /$ day.

The study was conducted before and after implementation of the revised hypoglycemia algorithm with dextrose gel: $05 / 2014$ to $10 / 2014$ and $11 / 2014$ to $04 / 2015$. We included infants at risk for neonatal hypoglycemia, with the following criteria: infants $<37$ weeks of age, SGA and LGA, maternal diabetes, cord $\mathrm{pH}<7.10$, cord base excess less than $-10 \mathrm{mEq} / \mathrm{l}$, as well as maternal treatment with $\beta$-blockers (propranolol), $\beta$-sympathomimetics (e.g., terbutaline), oral hypoglycemic agents (e.g., metformin, glyburide), indomethacin, or nifedipine. These infants were asymptomatic, 35 weeks PMA or greater and $<48 \mathrm{~h}$ old. We excluded infants with symptomatic hypoglycemia and congenital malformations. If hypoglycemia resolved with dextrose gel but recurred within $48 \mathrm{~h}$ of postnatal age, therapy with dextrose gel could be repeated twice (to a maximum of 3 doses during the entire 48-hour period) (fig. 1). We did not include infants older than $48 \mathrm{~h}$ as they are at risk for persistent hypoglycemic disorders, and, also, the lower limit for the glucose threshold in these infants is $60 \mathrm{mg} /$ dl [9].

Transfer to the NICU for IV dextrose was considered treatment failure and was the primary outcome. Secondary outcomes included exclusive breastfeeding rates at discharge and cost savings associated with the protocol for dextrose gel.

The physician and hospital costs were obtained from the University at Buffalo, Department of Pediatrics practice plan and the Finance Department of the Women and Children's Hospital and Millard Fillmore Suburban Hospital.

\section{Analysis}

Categorical data were analyzed using the $\chi^{2}$ test or Fisher's exact test as appropriate. Continuous data were analyzed using the independent sample t test or the Wilcoxon-MannWhitney test. Absolute risk reduction and the number needed to treat were calculated with confidence intervals (CI). For statistical significance, the probability was set at $<5 \%$. Analysis was performed with SPSS 22 (IBM, New York, N.Y., USA) and GraphPad (GraphPad Software Inc., La Jolla, Calif., USA)

\section{Results}

Before and after Dextrose Gel Implementation

During the pre- and post-dextrose gel protocol implementation epochs, there was no difference in the number of live births, asymptomatic hypoglycemic infants, infants of diabetic 
Table 1. Characteristics of asymptomatic hypoglycemic infants before and after the implementation of oral dextrose gel

\begin{tabular}{|c|c|c|c|}
\hline Characteristics & $\begin{array}{l}\text { May 1, 2014, to } \\
\text { October 31, } 2014 \\
\text { (pre-dextrose gel epoch) }\end{array}$ & $\begin{array}{l}\text { November 1, 2014, to } \\
\text { April 30, } 2015 \\
\text { (post-dextrose gel epoch) }\end{array}$ & $\mathrm{p}$ value \\
\hline Total live births & 2,937 & 2,654 & - \\
\hline Total asymptomatic infants with hypoglycemia & $248(8.4)$ & $250(9.4)$ & 0.20 \\
\hline $\begin{array}{l}\text { Improved with feeds in the pre-dextrose gel epoch/feeds }+ \text { dextrose gel in } \\
\text { the post-dextrose gel epoch }\end{array}$ & 144 (58) & $184(74)$ & $<0.01$ \\
\hline Transferred to the NICU for IV dextrose & $104(42)$ & $66(26)$ & $<0.01$ \\
\hline Maternal diabetes & $74(30)$ & $70(28)$ & 0.69 \\
\hline LGA & $48(20)$ & $33(13)$ & 0.06 \\
\hline SGA & $34(14)$ & $37(15)$ & 0.80 \\
\hline Exclusive breastfeeding at hospital discharge & $48(19)$ & $70(28)$ & 0.03 \\
\hline Overall total admissions to the NICU for all indications & $503(17)$ & $454(17)$ & 1.0 \\
\hline Number of babies transferred from the NBN to the NICU for hypoglycemia & $104(21)$ & $66(14)$ & 0.01 \\
\hline
\end{tabular}

Figures in parentheses are percentages.

mothers, SGA or LGA infants. Furthermore, during the two periods, there was no difference in total admissions to the NICU (table 1). However, the number of infants transferred from the NBN to the NICU for IV dextrose therapy significantly decreased with the implementation of the revised hypoglycemia algorithm with dextrose gel (fig. 1).

The number of infants transferred from the NBN to the NICU for hypoglycemia decreased from 35/1,000 to 25/1,000 live births ( $p<0.01$ ). As a percentage of all NICU admissions, those with an indication of asymptomatic hypoglycemia decreased from 20.6 to $14.8 \%$. After introduction of dextrose gel, only $26 \%$ of asymptomatic hypoglycemic neonates in the NBN were transferred to the NICU for IV dextrose compared to $42 \%$ during the pre-dextrose gel epoch (feeds alone). The exclusive breastfeeding rates at hospital discharge improved significantly after introduction of dextrose gel (table 1).

\section{Characteristics of Infants Treated with Oral Dextrose Gel}

Infants who failed therapy with dextrose gel and required IV fluids were LGA, more likely to have been delivered by cesarean section and had lower blood glucose levels. The Apgar scores were similar between responders and nonresponders (table 2). As expected, therapy with dextrose gel resulted in significantly higher blood glucose levels compared to nonresponders. Following therapy with dextrose gel, the median glucose levels were $58 \mathrm{mg} / \mathrm{dl}$ (IQR 45-86). Following IV dextrose therapy, the median glucose levels were $64 \mathrm{mg} / \mathrm{dl}$ (IQR 45-120). The number of doses of dextrose gel required and the babies who responded with each dose are shown in figure 2. Seventy of the 184 responders required only one dose of dextrose gel (fig. 2), while 28 of the 66 infants who failed therapy required IV dextrose therapy after the first dose.

\section{Impact of Using Oral Dextrose Gel}

The absolute risk reduction for IV dextrose therapy after the introduction of dextrose gel was 15.54\% (95\% CI 7.32-23.76). The number needed to treat was 7 (95\% CI 4.2-13.7). The average length of stay for infants who required IV dextrose therapy was $7.3 \pm 4.3$ days. The average duration of IV dextrose therapy alone was $3.4 \pm 2.6$ days. The length of stay for neonates who responded to dextrose gel was $3.1 \pm 1.1$ days. With treatment success, the breastfeeding rates at discharge were higher among the responders. 
2016 The Author(s). Published by S. Karger AG, Base www.karger.com/bmh

Rawat et al:: Oral Dextrose Gel Reduces the Need for Intravenous Dextrose Therapy in Neonatal Hypoglycemia

Table 2. Characteristics of infants based on glycemic response to dextrose gel

\begin{tabular}{|c|c|c|c|}
\hline Characteristics & $\begin{array}{l}\text { Responders } \\
\text { (no IV fluids after } \\
\text { dextrose gel) }\end{array}$ & $\begin{array}{l}\text { Nonresponders } \\
\text { (IV fluids after } \\
\text { dextrose gel) }\end{array}$ & $\mathrm{p}$ value \\
\hline Total infants & $184(74)$ & $66(26)$ & - \\
\hline Maternal age, years & $29 \pm 6$ & $30 \pm 4$ & 0.21 \\
\hline Maternal BMI & $33.7 \pm 8$ & $35.6 \pm 8$ & 0.09 \\
\hline Maternal diabetes & $47(26)$ & $24(36)$ & 0.12 \\
\hline Birth weight, g & $2,905 \pm 1,130$ & $3,110 \pm 1,173$ & 0.21 \\
\hline Gestational age, weeks & $38.6 \pm 1.6$ & $38.6 \pm 1.3$ & 0.98 \\
\hline Median Apgar score at $5 \mathrm{~min}$ (IQR) & $9(9-9)$ & $9(9-9)$ & 1.0 \\
\hline LGA & $18(10)$ & $15(23)$ & 0.02 \\
\hline SGA & $44(24)$ & $22(33)$ & 0.15 \\
\hline Cesarean section & $70(38)$ & $35(53)$ & 0.04 \\
\hline Exclusive breastfeeding & $62(34)$ & $8(12)$ & $<0.01$ \\
\hline Blood glucose before the 1 st dose of dextrose gel, mg/dl & $39.7 \pm 6$ & $33.6 \pm 9$ & $<0.01$ \\
\hline Blood glucose $1 \mathrm{~h}$ after the $1 \mathrm{st}$ dose of dextrose gel, $\mathrm{mg} / \mathrm{dl}$ & $54.7 \pm 12.6$ & $46.3 \pm 10$ & $<0.01$ \\
\hline Physician and hospital charges per patient, USD & $5,037 \pm 853$ & $36,391 \pm 13,504$ & $<0.01$ \\
\hline
\end{tabular}

Figures in parentheses are percentages, unless otherwise indicated.

Table 3. Economic impact of using oral dextrose gel

\begin{tabular}{lc}
\hline Parameters & Mean cost \pm SD, \\
& USD \\
\hline Cost for a NICU admission to treat hypoglycemia with IV dextrose therapy & $31,820 \pm 13,272$ \\
Cost for a regular NBN admission & $4,415 \pm 819$ \\
Cost for treating hypoglycemia successfully with oral dextrose gel & $5,037 \pm 873$
\end{tabular}

\section{Economic Impact}

The cost (physician billing and hospital charges) of care for asymptomatic infants with hypoglycemia in the newborn nursery (with feeds \pm dextrose gel) and in the NICU with IV dextrose are shown in tables 2 and 3 . The implementation of a protocol incorporating dextrose gel resulted in an overall cost savings of USD 642,951 over the 6-month period (or USD 2,593 per patient with asymptomatic hypoglycemia).

\section{Discussion}

This is the first report of an implementation of dextrose gel therapy for asymptomatic hypoglycemia in late preterm and term neonates at a regional perinatal center in the United States. We were successful in reducing the number of neonates requiring IV dextrose therapy and admission to the NICU, increasing exclusive breastfeeding at discharge and reducing health care costs.

Infants who are persistently hypoglycemic (blood glucose level $<45 \mathrm{mg} / \mathrm{dl}$ ) and asymptomatic in spite of feeds were treated with IV dextrose therapy to maintain euglycemia. Constant glucose infusion through dextrose therapy maintains the blood sugar, after which it is a common practice to wean IV fluids. IV dextrose therapy can also cause a rapid increase in 
(c) 2016 The Author(s). Published by S. Karger AG, Basel www.karger.com/bmh

Rawat et al.: Oral Dextrose Gel Reduces the Need for Intravenous Dextrose Therapy in Neonatal Hypoglycemia

Fig. 2. Response to dextrose gel among 250 patients with asymptomatic hypoglycemia managed with the hypoglycemia protocol shown in figure 1. Seventy patients responded to the first dose of dextrose gel and did not require further therapy. Twentyeight patients had low glucose levels of $<25 \mathrm{mg} / \mathrm{dl}$ after the first dose of dextrose gel and were transferred to the NICU for IV dextrose treatment. 152 patients had glucose levels of $25-45 \mathrm{mg} / \mathrm{dl}$ and were treated with a second dose of dextrose gel. Response to the second and third dose of dextrose gel is also shown.

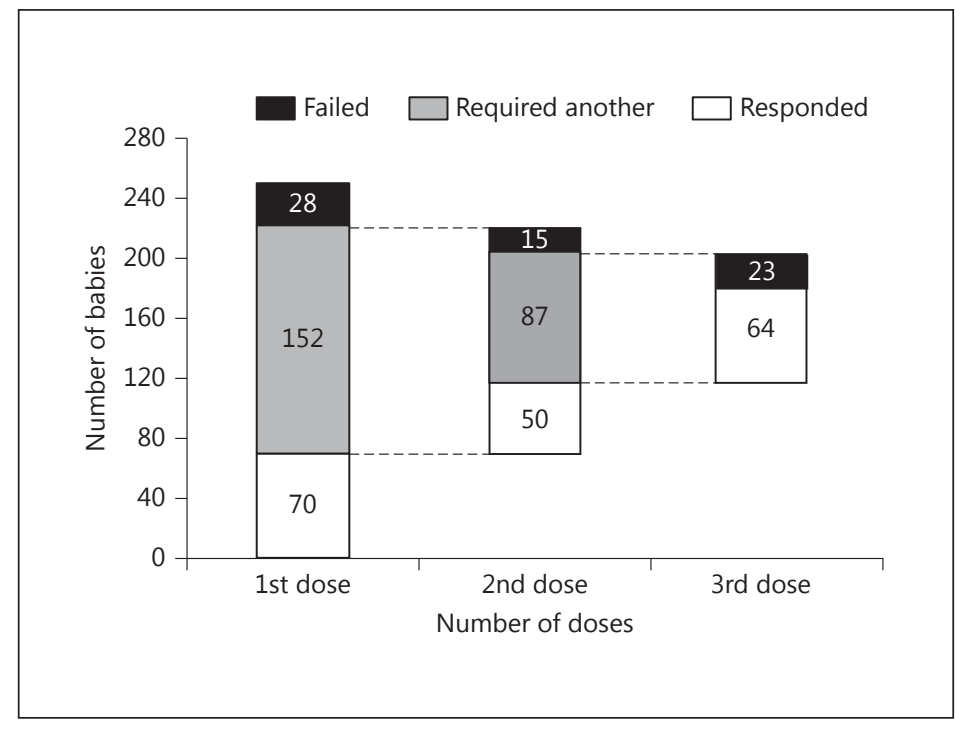

blood glucose level which can be potentially detrimental [10]. The increase in the blood glucose level after dextrose gel administration was within the physiological range, with no episodes of hyperglycemia.

Implementation of the revised hypoglycemia protocols included one grand rounds lecture followed by multiple in-service lectures by physician leaders, pharmacists and nurse educators. Compliance with the protocol increased rapidly during the first few days of implementation. The use of $40 \%$ dextrose gel was not associated with any adverse outcome or large fluctuations in blood glucose levels, which was consistent with the previous literature $[8,11]$.

Prior to the introduction of dextrose gel therapy, many of our infants were supplemented with formula for asymptomatic hypoglycemia due to concerns about lack of colostrum. This was based on the evidence that most term infants who were breastfed had blood sugar levels less than those of infants who were formula fed [12-14]. In the pre-intervention period, despite higher formula supplementation, the number of infants who required IV dextrose therapy was high compared to the post-dextrose gel epoch. After introducing dextrose gel and feeds together, the need for formula supplementation decreased, and exclusive breastfeeding rates at the time of discharge increased in these infants. A consistent result across the studies evaluating the use of dextrose gel is the improvement in exclusive breastfeeding rates [15]. Our study found similar results in that breastfeeding rates prior to discharge improved with implementation of dextrose gel and was higher in those infants who responded to therapy. Interestingly, this intervention resulted in an improvement in the Consumer Assessment of Healthcare Providers and Systems (CAHPS) score (Press Ganey ${ }^{\circledR}$ ). The percentage of mothers rating the hospital as 9-10 increased from 57.6\% (22nd percentile for the New York state peer group) in the pre-dextrose gel epoch to $68.4 \%$ (72nd percentile for the New York peer group). Similarly, the percentage of mothers answering 'definitely yes' for recommending this hospital increased from $61.7 \%$ (31st percentile) to $76.1 \%$ (83rd percentile). We acknowledge that factors other than dextrose gel administration may have played a role in this improvement.

Implementation of dextrose gel not only avoided the need for IV fluids but could also reduce the need for painful procedures such as venipuncture. Since oral glucose solution is used as comfort measures in the NICU, oral dextrose gel might also have mitigated the pain secondary to heel stick [16]. Infants who did not respond to dextrose gel therapy in our study had lower initial blood glucose levels and were more likely to be LGA compared to responders. 
We speculate that these infants probably experienced hyperinsulinemic hypoglycemia as opposed to transitional hypoglycemia in infants appropriate for gestational age.

Finally, the economic impact created by the introduction of oral dextrose gel therapy in reducing NICU admissions was quite significant. Although the number needed to treat was 7 , there were substantial reductions in physician and hospital charges (table 1). The majority of the infants who responded to oral dextrose gel therapy required only $1 \mathrm{dose}$.

Our study has several limitations. The comparison between feeds only and feeds and dextrose gel was not randomized but part of a pre-versus post-intervention study. In addition, during the pre-intervention period, data were collected retrospectively using the electronic medical record. After introduction of the dextrose gel, nurses and nurse practitioners recorded data in a prospective manner as part of this quality improvement project. Our initial blood glucose levels were obtained using a point-of-care glucometer which was later confirmed by the laboratory on a stat sample if the point-of-care blood sugar level was $<45 \mathrm{mg} / \mathrm{dl}$. Unlike many institutions, our nursery does not provide IV glucose, and a need for parenteral fluids necessitates the transfer to the NICU and escalates the health care cost. Our economic assessment does not cover the impact of breastfeeding. Finally, we did not have the follow-up data to see the breastfeeding rates after discharge.

To conclude, therapy with dextrose gel can be effectively incorporated into the hypoglycemia protocol for asymptomatic late preterm and term infants. Our study confirms the findings of previous studies that dextrose gel is a less expensive alternative therapy that can reduce the need for IV dextrose therapy, increase breastfeeding rates and improve parent satisfaction probably due to maternal infant bonding. A future trial, such as the hypoglycemia prevention with oral dextrose gel (ACTRN 12614001263684) [17], will shed light on the economic impact of this therapy. Large randomized control trials (NCT02523222 and ACTRN12613000322730) are being planned and will give us further insight into dextrose gel therapy for asymptomatic hypoglycemia and neurodevelopmental outcomes.

\section{Acknowledgement}

We thank the parents, our nurses, practitioners and pharmacists for their cooperation during the implementation of this protocol. They were an integral part of this process improvement. We would like to thank Mr. David Lewandowski and Dr. Valerie Elberson for the video of their daughter Elise Claire Lewandowski demonstrating the administration of oral dextrose gel. We thank Ms. Meredith Gedraitis and Ms. Gwen Spann for their assistance in providing health care cost data. Dr. Lakshminrusimha received NICHD grant No. 5 R01 HD072929.

\section{Disclosure Statement}

The authors have no conflicts of interest to disclose. 


\section{References}

1 Burns CM, Rutherford MA, Boardman JP, Cowan FM: Patterns of cerebral injury and neurodevelopmental outcomes after symptomatic neonatal hypoglycemia. Pediatrics 2008;122:65-74.

$>2$ Dalgic N, Ergenekon E, Soysal S, Koc E, Atalay Y, Gücüyener K: Transient neonatal hypoglycemia - long-term effects on neurodevelopmental outcome. J Pediatr Endocrinol Metab 2002;15:319-324.

-3 McKinlay CJ, Alsweiler JM, Ansell JM, Anstice NS, Chase JG, Gamble GD, Harris DL, Jacobs RJ, Jiang Y, Paudel N, Signal M, Thompson B, Wouldes TA, Yu TY, Harding JE, Group CS: Neonatal glycemia and neurodevelopmental outcomes at 2 years. N Engl J Med 2015;373:1507-1518.

-4 Cornblath M, Hawdon JM, Williams AF, Aynsley-Green A, Ward-Platt MP, Schwartz R, Kalhan SC: Controversies regarding definition of neonatal hypoglycemia: suggested operational thresholds. Pediatrics 2000;105:11411145.

5 March of Dimes Perinatal Data Center. Special care nursery admissions. 2011. https://www.marchofdimes. org/peristats/pdfdocs/nicu_summary_final.pdf

-6 Committee on Fetus and Newborn, Adamkin DH: Postnatal glucose homeostasis in late-preterm and term infants. Pediatrics 2011;127:575-579.

-7 Slama G, Traynard PY, Desplanque N, Pudar H, Dhunputh I, Letanoux M, Bornet FR, Tchobroutsky G: The search for an optimized treatment of hypoglycemia. Carbohydrates in tablets, solutin, or gel for the correction of insulin reactions. Arch Intern Med 1990;150:589-593.

-8 Weston PJ, Harris DL, Battin M, Brown J, Hegarty JE, Harding JE: Oral dextrose gel for the treatment of hypoglycaemia in newborn infants. Cochrane Database Syst Rev 2016;5:CD011027.

-9 Thornton PS, Stanley CA, De Leon DD, Harris D, Haymond MW, Hussain K, Levitsky LL, Murad MH, Rozance PJ, Simmons RA, Sperling MA, Weinstein DA, White NH, Wolfsdorf JI, Pediatric Endocrine Society: Recommendations from the pediatric endocrine society for evaluation and management of persistent hypoglycemia in neonates, infants, and children. J Pediatr 2015;167:238-245.

10 Ennis K, Dotterman H, Stein A, Rao R: Hyperglycemia accentuates and ketonemia attenuates hypoglycemiainduced neuronal injury in the developing rat brain. Pediatr Res 2015;77:84-90.

11 Harris DL, Weston PJ, Signal M, Chase JG, Harding JE: Dextrose gel for neonatal hypoglycaemia (the sugar babies study): a randomised, double-blind, placebo-controlled trial. Lancet 2013;382:2077-2083.

12 Deshpande S, Ward Platt M: The investigation and management of neonatal hypoglycaemia. Semin Fetal Neonatal Med 2005;10:351-361.

13 Hay WW Jr, Raju TN, Higgins RD, Kalhan SC, Devaskar SU: Knowledge gaps and research needs for understanding and treating neonatal hypoglycemia: workshop report from Eunice Kennedy Shriver National Institute of Child Health and Human Development. J Pediatr 2009;155:612-617.

$\checkmark 14$ Rozance PJ, Hay WW: Hypoglycemia in newborn infants: features associated with adverse outcomes. Biol Neonate 2006;90:74-86.

-15 Stewart CE, Sage EL, Reynolds P: Supporting 'baby friendly': a quality improvement initiative for the management of transitional neonatal hypoglycaemia. Arch Dis Child Fetal Neonatal Ed 2016;101:F344-F347.

16 American Academy of Pediatrics Committee on Fetus, Newborn; American Academy of Pediatrics Section on Surgery; Canadian Paediatric Society Fetus and Newborn Committee, Batton DG, Barrington KJ, Wallman C: Prevention and management of pain in the neonate: an update. Pediatrics 2006;118:2231-2241.

17 Harding JE, Hegarty JE, Crowther CA, Edlin R, Gamble G, Alsweiler JM: Randomised trial of neonatal hypoglycaemia prevention with oral dextrose gel (hPOD): study protocol. BMC Pediatr 2015;15:120. 